\title{
Examination of The Cevimeline Hydrochloride Hydrate Concomitant Hachiazule and Xylocaine Gargle Therapy
}

\author{
Hiroshi Mese $^{1)}$, Katsushi Furuno ${ }^{2)}$, Akira Sasaki ${ }^{1)}$ \\ 1) Department of Oral and Maxillofacial Surgery, Biopathological Science, Graduate School of Medicine, Dentistry and Pharmaceutical \\ Sciences, Okayama University Graduate Schools \\ 2) Department of Hospital Pharmacy, Okayama University Medical and Dental School
}

\begin{abstract}
A gargling treatment by Hachiazele and Xylocaine is useful for a stomatitis treatment of the oral cancer patient with chemotherapy and/or radiotherapy. However, there is a little improvement of dry mouth. Aiming at improvement of dry mouth for the oral cancer patient with chemotherapy and/or radiotherapy, we reviewed the cevimeline hydrochloride hydrate concomitant Hachiazule and Xylocaine gargling therapeutic safety. Homeostasis for ten days of cevimeline hydrochloride hydrate was confirmed in all condition by an incompatibility examination. It was significant, and the group of cevimeline hydrochloride hydrate combination elevated salivation dosage in comparison with a non-concomitant group. In addition, the side effect did not accept both groups together. As for the cevimeline hydrochloride hydrate combination Haciazule and Xylocaine gargling treatment, it was thought that beneficial gargling was therapeutic for dry mouth of the oral cancer patient with chemotherapy and/or radiotherapy.
\end{abstract}

\section{Introduction}

Oral mucositis is a frequent complication of cytoreductive cancer chemotherapy and radiotherapy. In many patients, it is associated with considerable pain, and thus, can significantly impair quality of life, in neutropenic patients with cancer, mucositis, represents a clinically significant risk factor for sepsis ${ }^{1)}$. Futhermore, in some patients, it becomes a dose-limiting toxicity, slowing or preventing continuation of selected cancer therapies, including accelerated fractionation and hyperfractionation in radiotherapy and interventions that combine chemotherapy and radiotherapy ${ }^{2}$.

A gargling treatment by Hachiazele and Xylocaine is useful for a stomatitis treatment of the oral cancer patient with chemotherapy and/or radiotherapy. However, there is a little improvement of dry mouth.

\section{Purpose}

Aiming at improvement of dry mouth for the oral cancer patient with chemotherapy and/or radiotherapy, we reviewed the cevimeline hydrochloride hydrate concomitant Hachiazule and Xylocaine gargling therapeutic safety.

\section{Methods}

1. It dissolves in distilled water $450 \mathrm{ml}$ at Hachiazule $10 \mathrm{~g}, 4 \%$ Xylocaine $5 \mathrm{ml}$, cevimeline hydrochloride hydrate $270 \mathrm{mg}$. The cevimeline hydrochloride hydrate incompatibility examination measured appearance after $0,1,2,5$ and 10 days and content cevimeline hydrochloride hydrate in cold place $\left(4^{\circ} \mathrm{C}\right)$, room temperature $\left(25^{\circ} \mathrm{C}\right)$ and high temperature place $\left(40^{\circ} \mathrm{C}\right)$.).

2. Examination intended for 20 normal volunteers (a group of A: cevimeline hydrochloride hydrate concomitant Hachiazule and Xylocaine, a group of B: Hachiazule and Xylocaine) and measured quantity of resting saliva after gargling 30, 60, 120 minutes later.

\section{Results}

1. Homeostasis for ten days of cevimeline hydrochloride hydrate was confirmed in all condition by an incompatibility examination.
2. It was significant, and the group of cevimeline hydrochloride hydrate combination (a group of A) elevated salivation dosage in comparison with a non-concomitant group (a group of B) $(p<0.05)$ (Fig.1,2). In addition, the side effect did not accept both groups together.

\section{Discussion}

Recently, cevimeline hydrochloride hydrate has been clinically applied to patients with Sjögren's syndrome for the treatment of xerostomia. Oral doses of cevimeline hydrochloride hydrate significantly improved subjective symptoms of dry mouth and dry eyes, and increased salivary flow ${ }^{3}$. However, adverse effects were frequently reported, such as nausea and abdominal pain. Unless properly managed, these adverse effects would negatively affect the continued administration cevimeline hydrochloride hydrate ${ }^{4}$.

As for the cevimeline hydrochloride hydrate combination Haciazule and Xylocaine gargling treatment, it was thought that beneficial gargling was therapeutic for dry mouth of the oral cancer patient with chemotherapy and/or radiotherapy. In addition, Hachiazule, which I used together this time, is combination of azulene and sodium bicarbonate. There is a little bitter taste and can do drainage without resistance by combination with cevimeline hydrochloride hydrate, too. I think that gargling of 2 agents except Xylocaine is effective for dry mouth without chemotherapy and/ or radiotherapy in oral cancer patients.

\section{Acknowledgement}

This work was supported in part by the Nippon Kayaku Co. Ltd.

\section{References}

1. Eliting L, Cooksley C, Chambers M, Cantor S, Manzullo E, Rubenstein T. The burdens of cancer therapy: clinical and economic outcomes of chemotherapy-induced mucositis. Cancer 98: 1531-1539, 2003

2. Sonis ST, Elting LS, Peterson DE, Schubert M, Hauer-Jensen M, Bekele BN, Raber-Durlacher J, Donnelly JP, Rubenstein 
J.Hard Tissue Biology.14(2)Proceeding,2005

EB. Perspectives on cancer therapy-induced mucosal injury. Cancer 100: 1995-2011, 2004

3. Fife RS, Chas WF, Dore RK, Wiesenhutter CW, Lockhart $\mathrm{PB}$, Tindal E, Suen JY. Cevimeline for the treatment of xerostomia in patients with Sjögren syndrome. Arch Intern Med 162:1293-1300, 2002

4. Takagi Y, Kimura T, Nakamura T. Cevimeline gargle for the treatment of xerostomia in patients with Sjögren's syndrome. Ann Rheum Dis 63:749, 2004

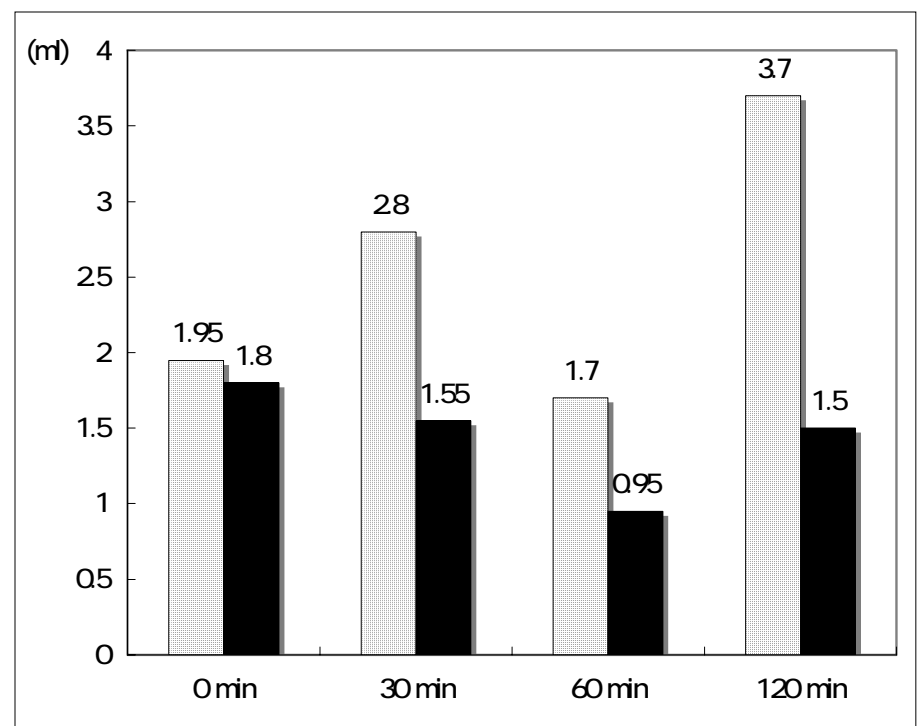

A group: cevimeline hydrochloride hydrates concomitant Hachiazule and Xylocaine.

B group: Hachiazule and Xylocaine

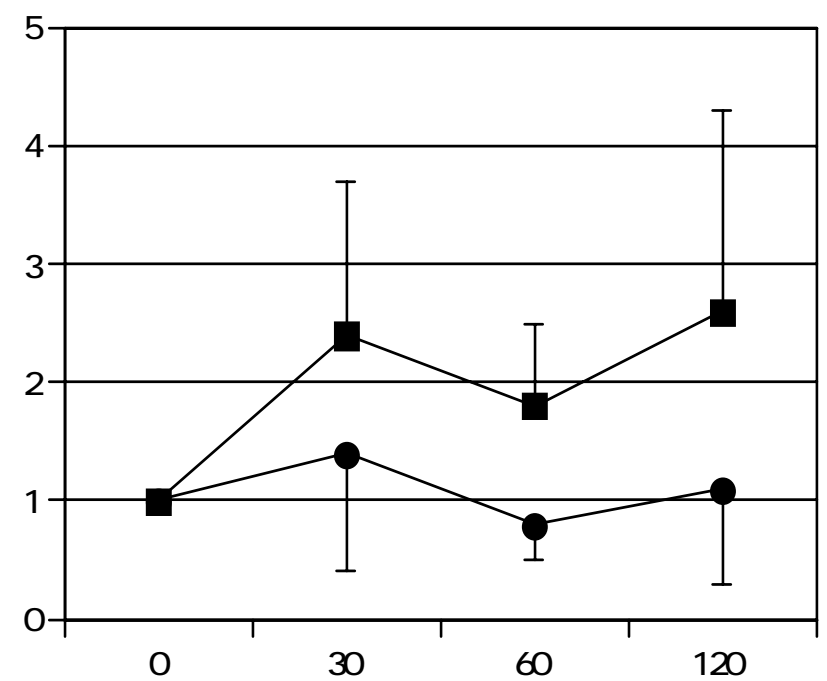

A group: cevimeline hydrochloride hydrates concomitant Hachiazule and Xylocaine B group: Hachiazule and Xylocaine 